\title{
Spatial prediction of landslide hazard at the Luxi area (China) using support vector machines
}

\begin{abstract}
The main objective of this study is to investigate the potential application of GIS-based Support Vector Machines (SVM) with four kernel functions, i.e., radial basis function (RBF), polynomial (PL), sigmoid (SIG), and linear (LN) for landslide susceptibility mapping at Luxi city in Jiangxi province, China. At the first stage of the study, a landslide inventory map with 282 landslide locations was identified using aerial photographs, satellite images, and field surveys. Of this, $70 \%$ of the landslides (196 landslide locations) are used as a training dataset and the rest (86 landslide locations) were used as the validation dataset. Then, 15 landslide conditioning factors were prepared, i.e., altitude, aspect, slope, stream power index (SPI), topographic wetness index (TWI), sediment transport index (STI), plan curvature, profile curvature, distance from river, distance from road, distance from fault, lithology, land use, NDVI, and rainfall. Using these conditioning factors, landslide susceptibility indexes were calculated using SVM with the four kernel functions. Subsequently, the results were exported and plotted in ArcGIS and four landslide susceptibility maps were produced. The four susceptibility maps were validated and compared using the landslide locations and the success rate and prediction rate methods. The validation results showed that success rates for the four SVM models are $82.0 \%$ (RBF), $83.0 \%$ (PL), $45.0 \%$ (SIG), and $70.0 \%$ (LN). The prediction rates for the four SVM models are $81.0 \%$ (RBF), $71.0 \%$ (PL), $40.0 \%$ (SIG), and LN $63.0 \%$ (SIG). The result shows that the RBF-SVM model has the highest overall performance. The produced susceptibility maps may be useful for general land-use planning in landslides.
\end{abstract}

Keyword: Landslide susceptibility; Support vector machine (SVM); Geographical information systems (GIS); Remote sensing; Luxi; China 